\begin{tabular}{|c|l|}
\hline Title & Paramagnetic instability of small topological superconductors \\
\hline Author(s) & Suzuki, Shu-Ichiro; A sano, Y asuhiro \\
\hline Citation & $\begin{array}{l}\text { Physical Review B, 89(18), 184508-1-184508.7 } \\
\text { https://doi.org/10.1103/PhysRevB.89.184508 }\end{array}$ \\
\hline Issue Date & 201405-01 \\
\hline Doc URL & http://hdl.handle.net/2115/57010 \\
\hline Rights & @2014 A merican Physical Society \\
\hline Type & article \\
\hline File Information & PhysRevB89 184508.pdf \\
\hline
\end{tabular}

Instructions for use 


\title{
Paramagnetic instability of small topological superconductors
}

\author{
Shu-Ichiro Suzuki ${ }^{1}$ and Yasuhiro Asano ${ }^{1,2}$ \\ ${ }^{1}$ Department of Applied Physics, Hokkaido University, Sapporo 060-8628, Japan \\ ${ }^{2}$ Center for Topological Science \& Technology, Hokkaido University, Sapporo 060-8628, Japan \\ (Received 26 December 2013; revised manuscript received 23 March 2014; published 14 May 2014)
}

\begin{abstract}
The diamagnetism is an essential property of all superconductors. However, we will show that small topological (or unconventional) superconductors can be intrinsically paramagnetic by solving the quasiclassical Eilenberger equation and the Maxwell equation self-consistently on two-dimensional superconducting disks in weak magnetic fields. Because of the topologically nontrivial character of the wave function, the unconventional superconductors host the zero-energy surface Andreev bound states, which always accompany so-called odd-frequency Cooper pairs. The paramagnetic property of the odd-frequency pairs explains the paramagnetic response of the disks at low temperature.
\end{abstract}

DOI: 10.1103/PhysRevB.89.184508

PACS number(s): 73.20.At, 73.20.Hb

\section{INTRODUCTION}

The Meissner effect is a fundamental property of superconductors as shown in standard textbooks [1]. The response of superconductors is usually diamagnetic because a superconductor excludes weak enough magnetic fields from its interior. The anomalous paramagnetic response, however, has been observed in small disks of metallic superconductor [2,3], small high- $T_{c}$ compounds [4-6], and mesoscopic proximity structures $[7,8]$. The spatial inhomogeneity of the magnetic property is a key feature to realize the paramagnetic phase. In metallic superconductors, the inhomogeneous distribution of magnetic fields [9] and the formation of giant vortex are responsible for the paramagnetic Meissner effect PME [10]. The presence of the $\pi$ junctions is also pointed out as an origin of PME in a network of Josephson junction [11]. In unconventional superconductors (USs), on the other hand, an experiment [6] has shown the decrease of the pair density with decreasing temperature, which suggests a peculiar mechanism of the PME unique to the USs. As a result of the topological nature in the wave function, the USs have the topologically protected surface Andreev bound states (ABSs) at the zero energy [12-16]. So far, theoretical studies have shown that the magnetic response at the (110) surface of high- $T_{c}$ superconductor is nonlinear $[17,18]$ and paramagnetic [19-22] due to the ABSs. The paramagnetic response has been mainly explained in terms of the energetics of the ABSs. Weak magnetic fields shift the energy of the surface ABSs away from the Fermi level and decrease the total energy of superconductor, which leads to the paramagnetic response or the paramagnetic instability. However, there is an important open question: what carries the large paramagnetic supercurrent? By addressing this issue, we will conclude that the magnetic properties of USs are intrinsically inhomogeneous and that small USs can be paramagnetic at low temperature.

The electric current in equilibrium has two contributions, (i.e., $\boldsymbol{j}=\boldsymbol{j}_{\mathrm{pq}}+\boldsymbol{j}_{\mathrm{A}}$ ). The quasiparticle current $\boldsymbol{j}_{\mathrm{pq}}$ due to the spatial phase gradient of the wave function is paramagnetic, whereas $\boldsymbol{j}_{\mathrm{A}}=-n e^{2} \boldsymbol{A} / m c$ is diamagnetic. In a normal metal, $\boldsymbol{j}_{\mathrm{pq}}$ cancels $\boldsymbol{j}_{\mathrm{A}}$ because the phase of an electron is not rigid at all [23]. In a superconductor, on the other hand, the phase rigidity of superconductivity drastically suppress the spatial gradient of phase, which leads to $\boldsymbol{j}_{\mathrm{qp}}=0$. As a result, a superconductor shows the perfect diamagnetism. In contrast to excited quasiparticles above, the superconducting gap, the quasiparticles below the gap have the phase rigidity because they are the shadow of Cooper pairs. In fact, a normal metal attaching to a metallic superconductor shows the diamagnetic Meissner effect [24]. This phenomenon is explained by two different but equivalent pictures: the penetration of a Cooper pair into the normal metal (proximity effect) and the Andreev reflection of a quasiparticle below the gap. The appearance of the surface ABSs is a direct result of the coherent Andreev reflections of a quasiparticle at the Fermi level [25]. Therefore such phase-rigid quasiparticles at the ABS cannot carry the large paramagnetic current.

In this paper, we theoretically study the spatial distribution of magnetic fields and that of electric currents on small two-dimensional superconducting disks with unconventional pairing symmetry such as spin-singlet $d$ wave and spin-triplet $p$ wave. There are several $d$-wave superconductors in organic compounds and heavy fermionic materials in addition to high- $T_{c}$ cuprates. Recently, the effective Hamiltonian for superconducting states in nanowires [26,27] has shown to be unitary equivalent to that for $p_{x}$-wave superconducting states [28]. The simulation at least two-dimensional system is necessary to evaluate the magnetic susceptibility quantitatively because the $d$ and $p$-wave pair potentials are anisotropic in real space. We solve the Eilenberger equation for the quasiclassical Green function and the Maxwell equation for magnetic fields self-consistently. The self-consistency of pair potential and magnetic field is necessary to regularize the nonlinear property in the magnetic response [17,18]. The solution of the Green function near the disk edge shows the presence of the odd-frequency Cooper pairs. The oddfrequency pairs have paramagnetic property [29-31] because of their negative pair density. The calculated results of the magnetic susceptibility suggest the PME in small USs. We conclude that the odd-frequency Cooper pairs carry the large paramagnetic current and causes the paramagnetic response of small superconducting disks.

\section{FORMULATION}

Let us consider a superconducting disk in two-dimension as shown in Fig. 1, where $R$ is the radius of the disk. We assume 

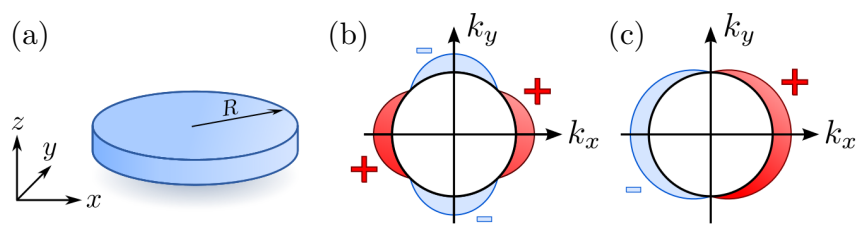

FIG. 1. (Color online) (a) The schematic figure of a superconducting disk. The pair potentials in momentum space are illustrated for the $d$ wave symmetry in (b) and for the $p$ wave symmetry in (c).

that the disk is in the clean limit and its surface is specular enough. To analyze the superconducting states in equilibrium, we solve the Eilenberger equation [32],

$$
\begin{gathered}
i \hbar v_{F} \hat{\boldsymbol{k}} \cdot \nabla_{\boldsymbol{r}} \breve{g}+[\check{H}, \breve{g}]=0, \\
\check{H}\left(\boldsymbol{r}, \boldsymbol{k}, i \omega_{n}\right)=\left[\begin{array}{cc}
\hat{\xi}\left(\boldsymbol{r}, \boldsymbol{k}, i \omega_{n}\right) & \hat{\Delta}(\boldsymbol{r}, \boldsymbol{k}) \\
\hat{\Delta}(\boldsymbol{r}, \boldsymbol{k}) & \hat{\xi}\left(\boldsymbol{r}, \boldsymbol{k}, i \omega_{n}\right)
\end{array}\right], \\
\check{g}\left(\boldsymbol{r}, \boldsymbol{k}, i \omega_{n}\right)=\left[\begin{array}{cc}
\hat{g}\left(\boldsymbol{r}, \boldsymbol{k}, i \omega_{n}\right) & \hat{f}\left(\boldsymbol{r}, \boldsymbol{k}, i \omega_{n}\right) \\
-\hat{\mathcal{L}}\left(\boldsymbol{r}, \boldsymbol{k}, i \omega_{n}\right) & -\hat{g}\left(\boldsymbol{r}, \boldsymbol{k}, i \omega_{n}\right)
\end{array}\right], \\
\hat{\xi}\left(\boldsymbol{r}, \boldsymbol{k}, i \omega_{n}\right)=i \omega_{n}+\left(e v_{F} / c\right) \boldsymbol{k} \cdot \boldsymbol{A}(\boldsymbol{r}),
\end{gathered}
$$

where $\boldsymbol{k}$ is the unit vector on the Fermi surface, $v_{F}$ is the Fermi velocity, $\omega_{n}=(2 n+1) \pi T$ is the Matsubara frequency, $n$ is an integer number, and $T$ is a temperature. In this paper, the symbol $\therefore$. represents $2 \times 2$ matrix structure in spin space and $\hat{\sigma}_{j}$ for $j=1-3$ are the Pauli matrices. The vector potential is denoted by $\boldsymbol{A}$ and the magnetic field $\boldsymbol{H}=\nabla \times \boldsymbol{A}$ is in the $z$ direction. We introduced a definition $\underset{\sim}{X}\left(\boldsymbol{r}, \boldsymbol{k}, i \omega_{n}\right) \equiv X^{*}(\boldsymbol{r},-$ $\left.\boldsymbol{k}, i \omega_{n}\right)$ for all functions $X$. The electric current is given by

$$
\boldsymbol{j}(\boldsymbol{r})=\frac{\pi e v_{F} N_{0}}{2 i} T \sum_{\omega_{n}} \int \frac{d \boldsymbol{k}}{2 \pi} \operatorname{Tr}\left[\check{T}_{3} \boldsymbol{k} \check{g}\left(\boldsymbol{r}, \boldsymbol{k}, \omega_{n}\right)\right],
$$

with $\check{T}_{3}=\operatorname{diag}\left[\hat{\sigma}_{0},-\hat{\sigma}_{0}\right]$, where $\hat{\sigma}_{0}$ is the identity matrix in spin space and $N_{0}$ is the density of state per spin at the Fermi level. We mainly consider the two equal-time pairing order parameters in two dimension: spin-singlet $d$-wave symmetry $\hat{\Delta}(\boldsymbol{r}, \theta)=\Delta(\boldsymbol{r}) \cos (2 \theta) i \hat{\sigma}_{2}$ and spin-triplet $p$-wave symmetry $\hat{\Delta}(\boldsymbol{r}, \theta)=\Delta(\boldsymbol{r}) \cos (\theta) \hat{\sigma}_{1}$, where $\theta$ is a directional angle with $k_{x}=\cos \theta$ and $k_{y}=\sin \theta$. The pair potentials are determined self-consistently from the gap equation

$$
\Delta(\boldsymbol{r}) i \hat{\sigma}_{\nu} \hat{\sigma}_{2}=\pi N_{0} g T \sum_{\omega_{n}} \int_{0}^{2 \pi} \frac{d \theta}{2 \pi} \hat{f}\left(\boldsymbol{r}, \theta, i \omega_{n}\right) V_{x}(\theta),
$$

where $x=s, p$ and $d$ indicate the pairing symmetry, $v=0$ and 3 for the spin-singlet and the spin-triplet order parameters, respectively. The coupling constant $g$ satisfies $\left\{N_{0} g\right\}^{-1}=$ $\ln \left(T / T_{c}\right)+\sum_{0 \leqslant n<\omega_{c} / 2 \pi T}(n+1 / 2)^{-1}$ with $T_{c}$ and $\omega_{c}$ being the transition temperature and the cutoff energy, respectively. The attractive potentials depends on the pairing symmetry $V_{x}(\theta)=$ $s_{x} \phi_{x}(\theta)$ with $s_{s}=1$ and $\phi_{s}(\theta)=1$ for $s$-wave symmetry, $s_{p}=2$ and $\phi_{p}(\theta)=\cos \theta$ for $p$-wave symmetry, and $s_{d}=2$ and $\phi_{d}(\theta)=\cos (2 \theta)$ for $d$-wave symmetry. The local magnetic susceptibility is defined by

$$
\chi_{m}(\boldsymbol{r})=\left(H(\boldsymbol{r})-H^{\mathrm{ext}}\right) /\left(4 \pi H^{\mathrm{ext}}\right),
$$

where $H^{\text {ext }}$ is the uniform external magnetic field in the $z$ direction. The susceptibility of the whole disk is calculated to be $\chi=\int d \boldsymbol{r} \chi_{m}(\boldsymbol{r}) /\left(\pi R^{2}\right)$. In the absence of spin-dependent potential, the spin structure of $\hat{\Delta}$ and that of $\hat{f}$ are always the same with each other. We use the standard Riccati parametrization [33-35] to solve the Eilenberger equation Eq. (1). To obtain numerical solutions of the Riccati type differential equation in closed disks, we apply a method discussed in Ref. [36]. An initial value at a certain place in the closed system is necessary to solve the Riccati equation. The obtained solution usually depends on the initial condition. However, when we solve the equation along the long enough classical trajectory, the effects of the initial condition is eliminated. In numerical simulation, we increase the length of the trajectory until solutions do not depend on the initial conditions. The vector potential $\boldsymbol{A}$ is obtained by solving the Maxwell equation $\nabla \times \boldsymbol{H}=(4 \pi / c) \boldsymbol{j}$ with Eq. (5). We calculate self-consistent solutions of the vector potential and pair potential by solving the Maxwell equation and the Eilenberger equation simultaneously. The anomalous Green function $\hat{f}\left(\boldsymbol{r}, \theta, i \omega_{n}\right)$ is originally defined by the two annihilation operators of two electrons consisting of a Cooper pair. Therefore $\hat{f}\left(\boldsymbol{r}, \theta, i \omega_{n}\right)$ must be antisymmetric under the interchange of the two electrons, which stems from the Fermi-Dirac statistics of electrons. Such fundamental relation is represented by

$$
\hat{f}\left(\boldsymbol{r}, \theta, i \omega_{n}\right)=-\left[\hat{f}\left(\boldsymbol{r}, \theta+\pi,-i \omega_{n}\right)\right]^{\mathrm{T}},
$$

where $\mathrm{T}$ represents the transpose of matrices.

\section{RESULTS}

The external magnetic field and the cut-off energy are fixed at $H^{\text {ext }}=0.001 H_{c_{1}}$ and $\omega_{c}=10 \Delta_{0}$, respectively. Here, $H_{c_{1}}=\hbar c /|e| \xi_{0}^{2}$ is the first critical magnetic field. The length is measured in units of $\xi_{0}=\hbar v_{F} / \Delta_{0}$ with $\Delta_{0}$ being the amplitude of the pair potential at $T=0$. The current density is normalized to $J_{0}=\hbar c /|e| \xi_{0}^{3}$. The characteristic length scale of the Maxwell equation is $\lambda_{L}=\left(4 \pi n e^{2} / m c^{2}\right)^{-1 / 2}$ and is a parameter in the numerical simulation. Throughout this paper, we use a unit of $k_{B}=1$.

In Fig. 2, we first show the calculated results of the local susceptibility (a) and the current density (b) for the $d$-wave superconducting disk, where we fix the parameters as $R=3 \xi_{0}, \lambda_{L}=3 \xi_{0}$, and $T=0.3 T_{c}$. We set $+x$ and $+y$ axes to be identical to (100) and (010) directions of the high- $T_{c}$ crystal. The central region of the disk is diamagnetic as usual, whereas the surfaces in the (110) and (110) directions are paramagnetic as shown in (a). The current density has the complex structure near the surface as shown in (b), where the arrow indicates the direction of current and its length represents the amplitude of current. Here we present the picture only for $x>0$ and $y>0$ in (b) because the results are fourfold symmetric due to the $d$-wave character of order parameter. The diamagnetic current flows at the edges in the (100) and (010) directions, whereas the paramagnetic current flows at the edges in the (110) direction. The vortexlike current profile can be seen near the surfaces because the two currents flow the opposite directions to each other. At the central region, on the other hand, only the diamagnetic current flows. Such magnetic properties are unique to unconventional superconductors. In 

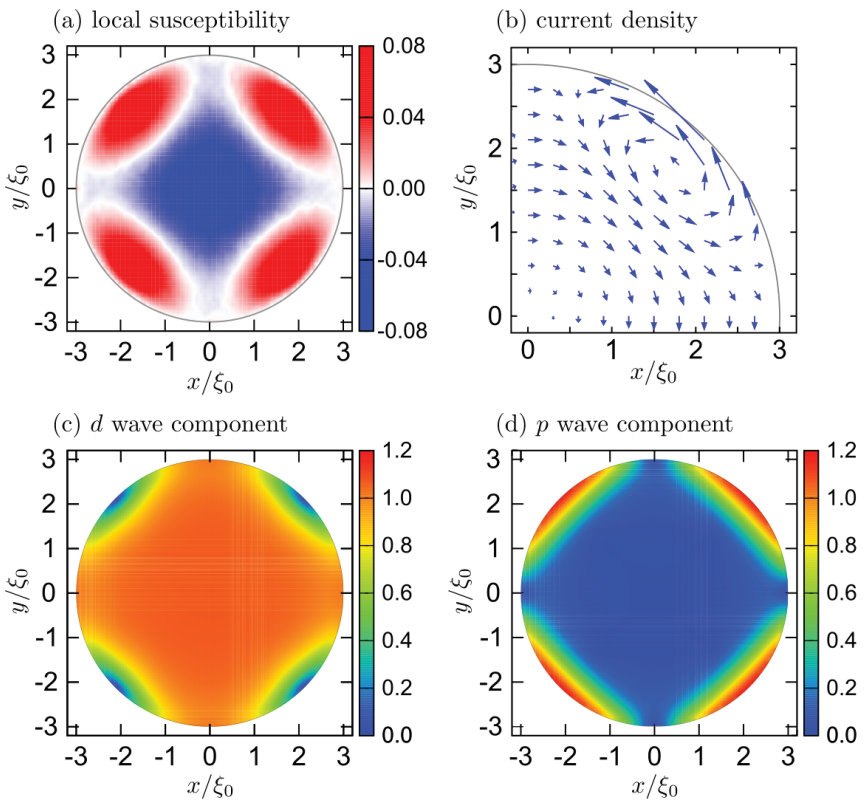

FIG. 2. (Color) (a) The local susceptibility and (b) the current density of the $d$-wave superconductor, where $R=3 \xi_{0}, \lambda_{L}=3 \xi_{0}$, $\omega_{c}=10 \Delta_{0}$, and $H^{\text {ext }}=0.001 H_{c 1}$. (c) The $d$-wave and (d) the $p$-wave components of the anomalous Green function.

$s$-wave case, the susceptibility is diamagnetic everywhere in the disk as show in the Appendix A.

The anomalous paramagnetic response is well explained by appearing the odd-frequency Cooper pairs. The anomalous Green function can be decomposed into $s-, p$-, and $d$-wave components by

$$
f_{x}\left(\boldsymbol{r}, i \omega_{n}\right) i \hat{\sigma}_{\nu} \hat{\sigma}_{2}=\int_{0}^{2 \pi} \frac{d \theta}{2 \pi} V_{x}(\theta) \hat{f}\left(\boldsymbol{r}, \theta, i \omega_{n}\right),
$$

for $x=s, p$, and $d$. Figure 2(c) shows the amplitude of the $d$-wave component at $\omega_{0}=\pi T$. The spatial profile of the order parameter is almost similar to that of (c). The $d$-wave component drastically suppresses in (110) and (11̄0) directions, which has been well known as a result of appearing of topologically protected Andreev surface bound states at the zero energy $[14,15]$. At the same time, the $p$-wave component of the anomalous Green function grows at the corresponding edges as shown in (d). The spin-singlet $p$-wave Cooper pairs must have the odd-frequency symmetry to satisfy Eq. (8). The breakdown of the translational symmetry at the surface mixes the even- and odd-parity components. The appearance of the Andreev surface bound states and that of the odd-frequency pairs are the two different faces of the same phenomenon. To have the zero-energy peak in the density of states, the frequency symmetry of Cooper pair must be odd [29,37,38]. The odd-frequency pairs have so called negative pair density [29], which leads to the paramagnetic instability as shown in Appendix B. Therefore we conclude that the paramagnetic current is carried by the induced odd-frequency Cooper pairs. Comparing the Figs. 2(b) with 2(d), the paramagnetic current flows at the regions where the odd-frequency Cooper pairs stay.

We have also obtained qualitatively the same results for a spin-triplet $p$-wave superconducting disk at $T=0.2 T_{c}$ as shown in Fig. 3, where the local magnetic susceptibility (a),
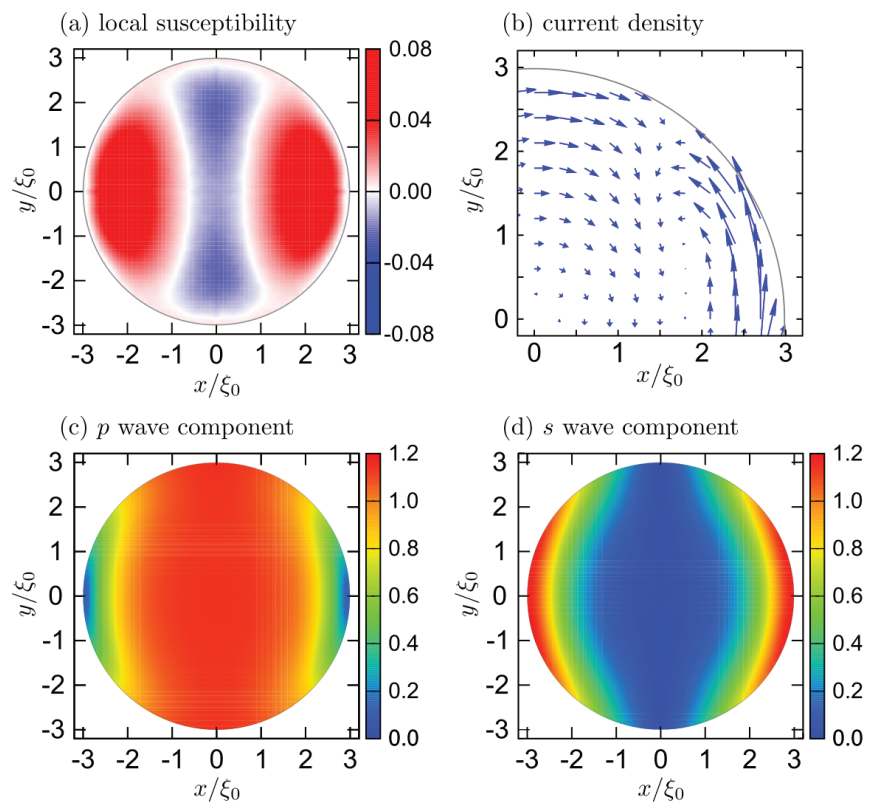

FIG. 3. (Color) (a) The local susceptibility and (b) the current density of the $p$-wave superconductor, where $R=3 \xi_{0}, \lambda_{L}=3 \xi_{0}$. (c) The $p$-wave and (d) the $s$-wave component of the anomalous Green function.

the current density (b), the $p$-wave component of $\hat{f}$ (c), and $s$-wave component of $\hat{f}(\mathrm{~d})$ are presented in the same manner as Fig. 2. The results in Fig. 3 show the twofold symmetry reflecting the $p$-wave order parameter. The surface bound states are appear at the (100) surfaces at which the $p$-wave component of the anomalous Green function is suppressed. Correspondingly, the $s$-wave component becomes large at the surfaces of (100) directions. The spin-triplet $s$-wave component belongs to the odd-frequency symmetry class according to Eq. (8). The main difference between Figs. 2 and 3 is the property of the surface ABS at the zero energy. In the spin-triplet $p$-wave disk, Majorana fermions appear at the surface [28]. From Figs. 2 and 3, we conclude that the magnetic property of unconventional superconductors are intrinsically inhomogeneous and can be paramagnetic because of the odd-frequency Cooper pairs at the surface.

The magnetic properties of superconductors strongly depends on the disk size because the odd-frequency pairs spatially localize near the surface limited by $\xi_{0}$ from the edge. Next, therefore, we discuss the relation between the magnetic property and the disk size. Figure 4 is the paramagneticdiamagnetic phase diagram of the $d$ - and $p$-wave superconducting disks, where the vertical axis is the paramagneticdiamagnetic crossover temperature $T_{p}$ and the horizontal one is the radius of superconducting disk $R$. The disk is paramagnetic $\chi>0$ at the temperatures below $T_{p}$. The results show that $T_{p}$ decrease with increasing the radius of the superconductor. As shown in Figs. 2 and 3, the paramagnetic area is limited to $\xi_{0}$ from the surface because odd-frequency pairs are confined there. On the other hand, the bulk area are diamagnetic because even-frequency pairs stay there. Roughly speaking, the relative area of staying the odd-frequency pairs to the whole area of disk qualitatively determines the magnetic response of the disk. Therefore the paramagnetic phase disappears in 


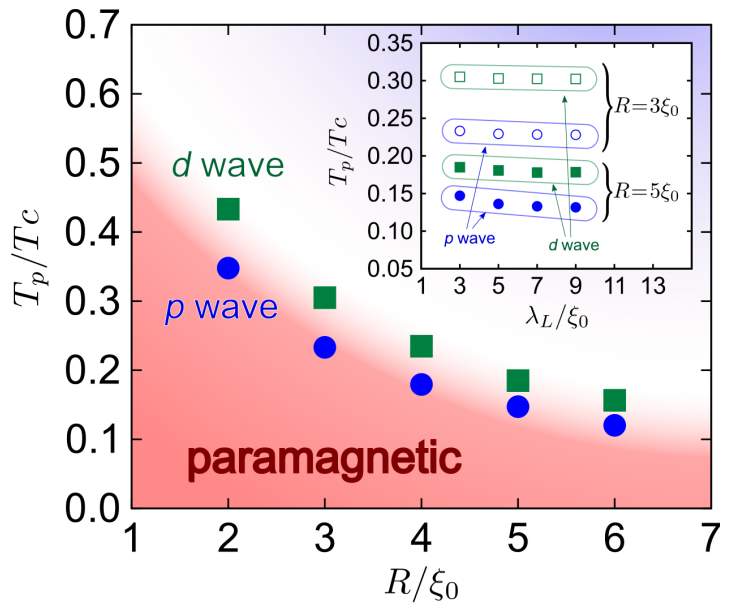

FIG. 4. (Color online) (a) The diamagnetic-paramagnetic phase diagram of superconducting disks for the $d$-wave (square) and $p$ wave (circle) pairing symmetry, where $\lambda_{L}=3 \xi_{0}, \omega_{c}=10 \Delta_{0}$. (b) The penetration length dependencies of the paramagnetic-diamagnetic crossover temperatures, where the square and circle symbols are the results for the $d$-wave and $p$-wave pairings, and the open and closed symbols are the results for the $R=3 \xi_{0}$ and $R=5 \xi_{0}$ superconducting disks, respectively.

large disks with $R \gg \xi_{0}$. because the contribution from the surface is negligible in large enough disks. This argument is supported by the $\lambda_{L}$ dependence of $T_{p}$ shown in the inset of Fig. 4, where open (filled) symbols represent the results for $R / \xi_{0}=3$ (5) and the circles (squares) are the results for $p(d)$ wave disks. The crossover temperature is totally insensitive to $\lambda_{L}$. To be paramagnetic, the larger disks require the stronger contribution from the odd-frequency Cooper pairs. The odd-frequency Cooper pairs energetically localize around the zero-energy [29]. The temperature smears effects of them on the magnetic response. Therefore $T_{p}$ decreases with increasing the disk size as shown in Fig. 4.

Finally, we discuss the susceptibility of whole superconducting disk as a function of temperature as shown in Fig. 5, where we fix the penetration depth at $\lambda_{L}=3 \xi_{0}$. The results for $d$ - and $p$-wave symmetries are presented in (a) and (b), respectively. The magnetic susceptibility just below $T_{c}$ is negative as usual. With decreasing temperature, the paramagnetic current due to the odd-frequency Cooper pairs increases. As a consequence, the susceptibility upturns at low temperature, which is qualitatively different from the susceptibility in the
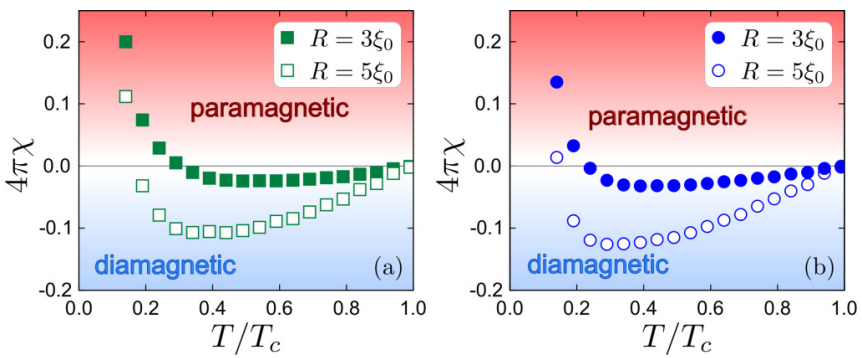

FIG. 5. (Color online) The temperature dependencies of the magnetic susceptibility for the (a) $d$-wave and (b) $p$-wave superconducting disks. $s$-wave case as shown in Appendix A. Below $T_{p}$, the paramagnetic odd-frequency Cooper pairs dominate the magnetic response of the superconductor. Therefore the dependence of the susceptibility on temperature shows the reentrant behavior as demonstrated in Fig. 5. In experiments, it is possible to measure the susceptibility as a function of temperature.

\section{DISCUSSION}

Our theoretical results may correlate to the measurement of the pair density at low temperature [6]. They measured the penetration depth $\lambda_{L}=\left(4 \pi n_{s} e^{2} / m c^{2}\right)^{-1 / 2}$ of a YBCO film on which (110) oriented internal surfaces are introduced by heavy-ion bombardment. They found that $\lambda$ first decreases with decreasing temperature from $T_{c}$ then increases at very low temperature. This results can be interpreted as a result of decreasing the pair density $n_{s}$ at low temperature. The odd-frequency pairs have the negative pair density. Thus the decrease of $n_{s}$ may suggest the increase of odd-frequency pair fraction. The experimental results on a high- $T_{c}$ superconductor are consistent with our theoretical results.

In real materials, the inelastic scatterings dephase the Cooper pairs and broaden the energy profile of the pairing functions. The inelastic mean free path also limits the size of disks in the phase diagram shown in Fig. 4. In $d$-wave superconductors, it has been shown that the surface roughness also broadens the zero-energy peak at the surface. In such situation, we infer that the roughness would suppress the paramagnetic effect. On the other hand in $p$-wave superconductors, the surface zero-energy peak is robust under the disordered potential. Therefore effects of surface roughness on the paramagnetic effect would be different in the two pairing symmetries. This is an important future issue.

The diamagnetism of superconductor is a result of gaining the condensation energy below the transition temperature. Therefore the paramagnetic superconducting states may be impossible in uniform thermodynamic limit. The paramagnetic phase in Fig. 4 can be considered as an unstable state and should disappear for large $R / \xi_{0}$. As shown in Figs. 2 and 3, the magnetic inhomogeneity is an intrinsic feature of unconventional superconductors. Such inhomogeneous property assists the appearance of the paramagnetic phase in small disks. Indeed, we confirm that the paramagnetic phase appears in two cooling processes: field cool and zero-field cool.

The spontaneously time-reversal symmetry (TRS) breaking states has been discussed in high- $T_{c}$ grains [42]. The subdominant component of order parameter near the surface breaks TRS. The results in Fig. 2 also indicates the TRS breaking superconducting state even when we simply assume the pure $d$-wave order parameter. We are thinking that the symmetry crossover from $d$ wave to TRS breaking $d+i s$ might be possible in small samples. To prove this, however, we need to compare the free-energy among possible symmetry states. This issue goes beyond the scope of this paper.

Odd-frequency pairs appear also in superconductor/ferromagnet proximity structures [38]. When oddfrequency pairs are dominant in the ferromagnet [39-41], the paramagnetic instability may lead to spontaneous current there [31]. 


\section{CONCLUSION}

In conclusion, we have theoretically studied the magnetic response of small unconventional superconducting disks by using the quasiclassical Green function method. We conclude that small unconventional superconductors can be paramagnetic at low temperature due to the appearance of odd-frequency Cooper pairs at their surface. The magnetic properties of unconventional superconductors are intrinsically inhomogeneous as a result of their topologically nontrivial nature. Our results show up such universal property of unconventional superconductivity.

\section{ACKNOWLEDGMENTS}

The authors are grateful to Y. Tanaka and S. Higashitani for useful discussion. This work was supported by the "Topological Quantum Phenomena" (No. 22103002) Grant-in Aid for Scientific Research on Innovative Areas from the Ministry of Education, Culture, Sports, Science and Technology (MEXT) of Japan.

\section{APPENDIX A: RESULTS FOR $s$-WAVE DISK}

We supply the calculated results for conventional spinsinglet $s$-wave superconductors. Figure 6 shows the calculated results of the local susceptibility (a) and the current density (b) for the $s$-wave superconducting disk, where we fix the parameters as $R=3 \xi_{0}, \lambda_{L}=3 \xi_{0}$, and $T=0.2 T_{c}$. Because of the isotropic property in the $s$-wave pair potential, the results are also isotropic in real space. Therefore we plot the results as a function of $x$ at $y=0$. The results in (a) show that the response is diamagnetic everywhere in the (a) local susceptibility

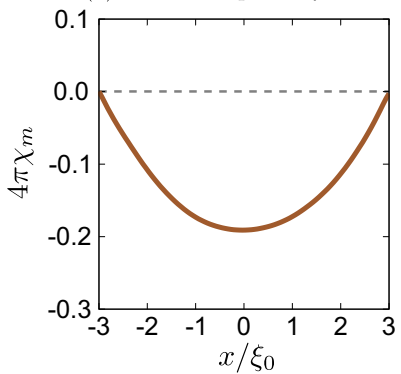

(c) $s$ wave component

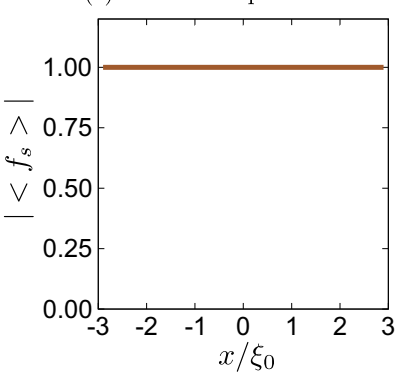

(b) current density
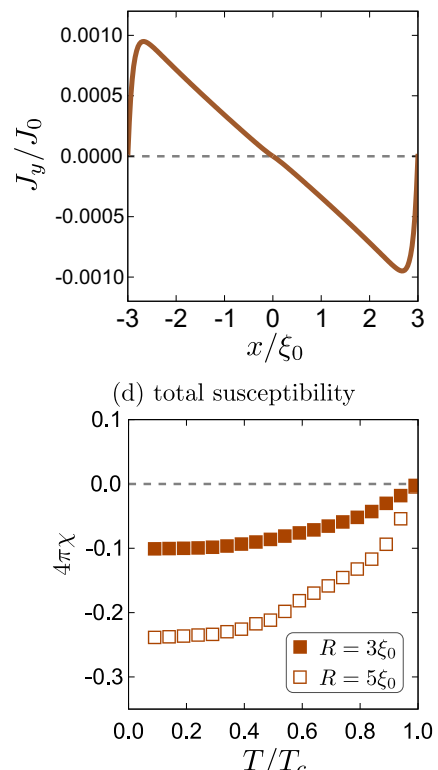

FIG. 6. (Color online) (a) The local susceptibility and (b) the current density of the $s$-wave superconductor, where $R=3 \xi_{0}, \lambda_{L}=$ $3 \xi_{0}, \omega_{c}=10 \Delta_{0}, H^{\mathrm{ext}}=0.001 H_{c 1}$ and $T=0.2 T_{c}$. (c) The $s$-wave component of the anomalous Green function. (d) The susceptibility vs temperature. disk. Correspondingly, the current profile in (b) suggests the usual Meissner screening current. The amplitude of the $s$-wave component of the anomalous Green function is almost uniform because $s$-wave superconductors are topologically trivial and do not host any surface states. The amplitude of the $p$-wave component is much smaller than that of $s$-wave one. The susceptibility of disk is plotted as a function of temperature in $(\mathrm{d})$. The susceptibility decreases monotonically with decreasing temperature, which is usually observed in experiments. Since the disk size is not much larger than $\lambda_{L}$, the perfect diamagnetism (i.e., $4 \pi \chi=-1$ ) is not archived.

\section{APPENDIX B: PARAMAGNETIC CURRENT DUE TO ODD-FREQUENCY PAIRS}

We discuss the contribution of odd-frequency pairs to the paramagnetic current within the linear response theory. Here we consider that the pair potential has a single component in spin space $\hat{\Delta}(\boldsymbol{r}, \boldsymbol{k})=\Delta(\boldsymbol{r}, \boldsymbol{k}) i \hat{\sigma}_{\nu} \hat{\sigma}_{2}$, where $v$ is one of $0-3$. In such case, the Eilenberger equation is reduces to a $2 \times 2$ matrix equation:

$$
i \hbar v_{F} \boldsymbol{k} \cdot \nabla_{\boldsymbol{r}} \hat{g}+[\hat{H}, \hat{g}]=0
$$

$$
\begin{gathered}
\hat{H}=\left[\begin{array}{cc}
i \omega_{n}+\frac{e v_{F}}{c} \boldsymbol{k} \cdot \boldsymbol{A} & i \Delta(\boldsymbol{r}, \boldsymbol{k}) \\
i \Delta(\boldsymbol{r}, \boldsymbol{k}) & -i \omega_{n}-\frac{e v_{F}}{c} \boldsymbol{k} \cdot \boldsymbol{A}
\end{array}\right], \\
\hat{g}\left(\boldsymbol{r}, \boldsymbol{k}, \omega_{n}\right)=\left[\begin{array}{cc}
g\left(\boldsymbol{r}, \boldsymbol{k}, \omega_{n}\right) & f\left(\boldsymbol{r}, \boldsymbol{k}, \omega_{n}\right) \\
s_{p} f\left(\boldsymbol{r}, \boldsymbol{k}, \omega_{n}\right) & -g\left(\boldsymbol{r}, \boldsymbol{k}, \omega_{n}\right)
\end{array}\right],
\end{gathered}
$$

where $s_{p}$ is 1 for even-parity order parameter and -1 for odd-parity one. The electric current is given by

$$
\boldsymbol{j}(\boldsymbol{r})=-2 i e v_{F} \pi N_{0} T \sum_{\omega_{n}} \int \frac{d \boldsymbol{k}}{S_{d}} \boldsymbol{k} g\left(\boldsymbol{r}, \boldsymbol{k}, \omega_{n}\right) .
$$

Here, $g$ is the normal Green function in the presence of the vector potential. In what follows, we estimate $g$ within the linear response of $\boldsymbol{A}$. In the Eilenberger equation, the vector potential formally shifts the energy. Thus the Green function can be expressed as

$$
g=g_{0}+\partial_{\omega_{n}} g_{0}\left(-i e v_{F} / c\right) \boldsymbol{k} \cdot \boldsymbol{A}
$$

within the linear response, where $g_{0}$ is the Green function at $\boldsymbol{A}=0$. In what follows, we omitted " 0 " from the subscript of the Green function for simplicity. By substituting the expression to Eq. (B3), we obtain

$$
\begin{aligned}
\boldsymbol{j}(\boldsymbol{r}) & =-\frac{n e^{2} \pi \boldsymbol{A}}{2 m c} T \sum_{\omega_{n}} \partial_{\omega_{n}}\left\langle g\left(\boldsymbol{r}, \omega_{n}\right)\right\rangle_{\boldsymbol{k}} \\
& =-\frac{n_{s} e^{2} \boldsymbol{A}}{m c}, \\
\frac{n_{s}}{n} & =\pi T \sum_{\omega_{n}}\left\langle\partial_{\omega_{n}} g\left(\boldsymbol{r}, \omega_{n}\right)\right\rangle_{\boldsymbol{k}} \\
& =\frac{1}{2} \int_{-\infty}^{\infty} d \omega\left\langle\partial_{\omega} g(\boldsymbol{r}, \omega)\right\rangle_{\boldsymbol{k}},
\end{aligned}
$$

where $n$ is the density of electrons in the normal state and $N_{0}$ is the density of states per spin at the Fermi level. The $\omega_{n}$ derivative of the Green function can be defined only at 
$T=0$. To discuss magnetic property of the anomalous Green function, we write the derivative of $g$ to

$$
\begin{array}{r}
\partial_{\omega} g(\boldsymbol{r}, \boldsymbol{k}, \omega)=\frac{1}{2}\left(f_{\mathrm{E}}^{2}-f_{\mathrm{O}}^{2}\right) \partial_{\omega} \ln \left(\frac{1+g}{1-g}\right), \\
f_{\mathrm{E}}\left(\boldsymbol{r}, \boldsymbol{k}, \omega_{n}\right)=\left.\frac{1}{2}\left(f+s_{p} \underset{\sim}{f}\right)\right|_{\left(\boldsymbol{r}, \boldsymbol{k}, \omega_{n}\right)}, \\
f_{\mathrm{O}}\left(\boldsymbol{r}, \boldsymbol{k}, \omega_{n}\right)=\left.\frac{1}{2}\left(f-s_{p} f\right)\right|_{\left(\boldsymbol{r}, \boldsymbol{k}, \omega_{n}\right)},
\end{array}
$$

where we have used the normalization condition in the Matsubara representation $g^{2}+s_{p} f f=1$ [43].

At $\boldsymbol{A}=0$, the Eilenberger equation is decomposed into three equations for the three components in the matrix structure,

$$
\begin{gathered}
\hbar v_{F} \boldsymbol{k} \cdot \nabla_{\boldsymbol{r}} g=2 \Delta f_{\mathrm{O}}, \\
\hbar v_{F} \boldsymbol{k} \cdot \nabla_{\boldsymbol{r}} f_{\mathrm{E}}=-2 \omega_{n} f_{\mathrm{O}}, \\
\hbar v_{F} \boldsymbol{k} \cdot \nabla_{\boldsymbol{r}} f_{\mathrm{O}}=2\left(\Delta g-\omega_{n} f_{\mathrm{E}}\right) .
\end{gathered}
$$

Here we note that the all functions are real when we delete the superconducting phase. For the uniform case, we obtain the solution

$$
g=\frac{\omega_{n}}{\sqrt{\omega_{n}^{2}+\Delta^{2}}}, \quad f=s_{p} f=f_{\mathrm{E}}=\frac{\Delta}{\sqrt{\omega_{n}^{2}+\Delta^{2}}},
$$

and $f_{\mathrm{O}}=0$. In the uniform bulk region, $f_{\mathrm{E}}$ is the source of the order parameter $\Delta$. Thus parity, spin, and frequency symmetries of $f_{\mathrm{E}}$ and those of $\Delta$ should be identical to each other. The contribution of $f_{\mathrm{E}}$ to the pair density must be positive in Eq. (B10) because the uniform superconductor is diamagnetic. The surface and the interface are source of the inhomogeneity in superconductor and mix the two orbital symmetry: even parity and odd parity. Nonzero spatial derivative in Eqs. (B13)-(B15) allows $f_{\mathrm{O}}$ component.

The component $f_{\mathrm{O}}$ is an odd function of $\omega_{n}$ as shown in Eq. (B13) because $g$ is always an odd function of $\omega_{n}$ due to a symmetry relationship $g\left(\boldsymbol{r}, \boldsymbol{k}, \omega_{n}\right)=-g^{*}\left(\boldsymbol{r}, \boldsymbol{k},-\omega_{n}\right)$. Equation (B14) indicates that the frequency symmetry of $f_{\mathrm{O}}$ are always opposite to those in $f_{\mathrm{E}}$. Therefore inhomogeneity induces $f_{\mathrm{O}}$ component which has the odd-frequency symmetry. Eq. (B10) tells us that the pair density of odd-frequency component is negative, which leads to the paramagnetic response.
[1] See, for example, M. Tinkham, Introduction to Superconductivity, 2nd ed. (McGraw-Hill, New York, 1996).

[2] D. J. Thompson, M. S. M. Minhaj, L. E. Wenger, and J. T. Chen, Phys. Rev. Lett. 75, 529 (1995).

[3] A. K. Geim, S. V. Dubonos, J. G. S. Lok, M. Henini, and J. C. Maan, Nature (London) 396, 144 (1998).

[4] W. Braunisch, N. Knauf, V. Kataev, S. Neuhausen, A. Grutz, A. Kock, B. Roden, D. Khomskii, and D. Wohlleben, Phys. Rev. Lett. 68, 1908 (1992).

[5] B. Schliepe, M. Stindtmann, I. Nikolic, and K. Baberschke, Phys. Rev. B 47, 8331 (1993).

[6] H. Walter, W. Prusseit, R. Semerad, H. Kinder, W. Assmann, H. Huber, H. Burkhardt, D. Rainer, and J. A. Sauls, Phys. Rev. Lett. 80, 3598 (1998).

[7] P. Visani, A. C. Mota, and A. Pollini, Phys. Rev. Lett. 65, 1514 (1990).

[8] A. C. Mota, P. Visani, A. Pollini, and K. Aupke, Physica B 197, 95 (1994).

[9] A. E. Koshelev and A. I. Larkin, Phys. Rev. B 52, 13559 (1995).

[10] V. V. Moshchalkov, X. G. Qiu, and V. Bruyndoncx, Phys. Rev. B 55, 11793 (1997).

[11] D. Dominguez, E. A. Jagla, and C. A. Balseiro, Phys. Rev. Lett. 72, 2773 (1994).

[12] L. J. Buchholtz and G. Zwicknagl, Phys. Rev. B 23, 5788 (1981).

[13] J. Hara and K. Nagai, Prog. Theor. Phys. 74, 1237 (1986).

[14] C. R. Hu, Phys. Rev. Lett. 72, 1526 (1994).

[15] Y. Tanaka and S. Kashiwaya, Phys. Rev. Lett. 74, 3451 (1995).

[16] M. Sato, Y. Tanaka, K. Yada, and T. Yokoyama, Phys. Rev. B 83, 224511 (2011); the topological number for two-dimensional $d$ and $p$-wave superconductors is defined in terms of the subgap wave functions at partial Brillouin zone in one dimension. Such characterization explain the dispersionless zero-energy bound states at their surface in the clean limit.

[17] S. K. Yip and J. A. Sauls, Phys. Rev. Lett. 69, 2264 (1992).

[18] A. Zare, T. Dahm, and N. Schopohl, Phys. Rev. Lett. 104, 237001 (2010).

[19] M. Fogelström, D. Rainer, and J. A. Sauls, Phys. Rev. Lett. 79, 281 (1997).

[20] Yu. S. Barash, M. S. Kalenkov, and J. Kurkijarvi, Phys. Rev. B 62, 6665 (2000).

[21] S. Higashitani, J. Phys. Soc. Jpn. 66, 2556 (1997).

[22] T. Löfwander, V. S. Shumeiko, and G. Wendin, Phys. Rev. B 62, R14653 (2000).

[23] A. A. Abrikosov, L. P. Gor'kov, and I. E. Dzyaloshinski, Methods of Quantum Field Theory in Statistical Physics (Dover, New York, 1975).

[24] W. Belzig, C. Bruder, and A. L. Fauchere, Phys. Rev. B 58, 14531 (1998).

[25] Y. Asano, Y. Tanaka, and S. Kashiwaya, Phys. Rev. B 69, 134501 (2004).

[26] R. M. Lutchyn, J. D. Sau, and S. Das Sarma, Phys. Rev. Lett. 105, 077001 (2010).

[27] Y. Oreg, G. Refael, and F. von Oppen, Phys. Rev. Lett. 105, 177002 (2010).

[28] Y. Asano and Y. Tanaka, Phys. Rev. B 87, 104513 (2013).

[29] Y. Asano, A. A. Golubov, Y. V. Fominov, and Y. Tanaka, Phys. Rev. Lett. 107, 087001 (2011).

[30] T. Yokoyama, Y Tanaka, and N Nagaosa, Phys. Rev. Lett. 106, 246601 (2011).

[31] S. Mironov, A. Mel'nikov, and A. Buzdin, Phys. Rev. Lett. 109, 237002 (2012).

[32] G. Eilenberger, Z. Phys. 214, 195 (1968). 
[33] N. Schopohl and K. Maki, Phys. Rev. B 52, 490 (1995).

[34] N. Schopohl, arXiv:cond-mat/9804064.

[35] M. Eschrig, Phys. Rev. B 80, 134511 (2009).

[36] Y. Nagai, K. Tanaka, and N. Hayashi, Phys. Rev. B 86, 094526 (2012).

[37] Y. Tanaka and A. A. Golubov, Phys. Rev. Lett. 98, 037003 (2007).

[38] F. S. Bergeret, A. F. Volkov, and K. B. Efetov, Phys. Rev. Lett. 86, 4096 (2001).
[39] Y. Asano, Y. Tanaka, and A. A. Golubov, Phys. Rev. Lett. 98, 107002 (2007).

[40] V. Braude and Yu. V. Nazarov, Phys. Rev. Lett. 98, 077003 (2007).

[41] M. Eschrig and T. Löfwander, Nat. Phys. 4, 138 (2008).

[42] A. M. Black-Schaffer, D. S. Golubev, T. Bauch, F. Lombardi, and M. Fogelström, Phys. Rev. Lett. 110, 197001 (2013).

[43] S. Higashitani, Phys. Rev. B 89, 184505 (2014). 